http://sciforum.net/conference/ece-1

Conference Proceedings Paper - Energies ,, Whither Energy Conversion? Present Trends, Current Problems and Realistic Future Solutions"

\title{
The Potential of Tandem Photovoltaic Solar Cells for Indoor Applications
}

\section{Ben Minnaert ${ }^{1, *}$ and Peter Veelaert ${ }^{1}$}

1 Faculty of Engineering and Architecture, Ghent University, Valentin Vaerwyckweg 1, B-9000 Gent, Belgium; E-Mails: Ben.Minnaert@UGent.be (B.M.); Peter.Veelaert@UGent.be (P.V.)

* Author to whom correspondence should be addressed; E-Mail: Ben.Minnaert@UGent.be (B.M.); Tel.: +32-9-243-2589.

Received: 13 January 2014 / Accepted: 13 March 2014 / Published: 14 March 2014

\begin{abstract}
Several studies have already determined the power potential of different types of photovoltaic solar cells for indoor applications. However, a detailed study whether or not the use of tandem solar cell (i.e. using two solar cells with different bandgaps in a row) is beneficial for indoor use is lacking. This paper attempts to fill this lacuna by comparing the power output of different tandem solar cells with single-junctions as reference. The comparisons are done by efficiency simulations of the solar cells and the light spectra of typical artificial light sources, i.e. a typical fluorescent tube, a high pressure sodium and metal halide lamp, a typical LED lamp and a common incandescent lamp, which are compared to the outdoor AM 1.5 spectrum as reference. More specifically, we study the influence of the energy levels of the bandgaps, not only for the usual monolithic configuration, but also for a stacked set-up. By determining the relative efficiency gain compared to single-junctions, we prove the limited usefulness of tandem solar cells for indoor applications.
\end{abstract}

Keywords: photovoltaic energy; solar cells; indoor applications; tandem cells.

\section{Introduction}

Nowadays, wireless communication networks focused towards indoor applications, use batteries as their source of energy. However, batteries have a limited lifetime and have to be replaced in due time. The lifetime of the battery is often the limiting factor for the lifetime of the device. Often, the cost of 
replacing the battery outweighs the cost of the device itself. Also from an environmental perspective, battery waste should be minimized if possible. The lifetime of the device can be extended if the device itself would be able to harvest energy from renewable resources in the environment. Photovoltaic (PV) solar energy can be an efficient natural energy source for indoor applications.

The different PV cells for applications on earth are rated by their power output under standard test conditions i.e. an illumination intensity of $10^{3} \mathrm{~W} / \mathrm{m}^{2}$ under the global AM 1.5G spectrum, at a cell temperature of $25{ }^{\circ} \mathrm{C}$. Although these conditions seldom appear at the same time, this characterization gives a reasonable guideline for comparing different solar cell types for outdoor conditions. However, the standard test conditions are not relevant for indoor applications. Typically, the light intensity under artificial lighting conditions found in offices and factories is less than $10 \mathrm{~W} / \mathrm{m}^{2}$ as compared to 100 $1000 \mathrm{~W} / \mathrm{m}^{2}$ under outdoor conditions, depending on the type of and the distance from the light source. Moreover, the spectrum can be totally different from the outdoor solar spectrum. The spectrum depends not only on the type of light source, but also on the presence of reflected and diffused light. Unfortunately, there are no international norms which determine the way of characterizing solar cells for indoor applications.

Different authors discussed [1-3] and measured [4-7] the spectral mismatch between the absorption of solar cells and the spectrum of indoor light sources, but mainly only for single-junction solar cells. In this paper we quantify on a fundamental level the gain that can be achieved by using tandem cells instead of single-junction cells for indoor applications. More specifically, we study the influence of the energy levels of the bandgaps of the tandem cell for different indoor illuminations, not only for the usual monolithic configuration, but also for a stacked set-up.

\section{Methodology}

The power conversion efficiency of a solar cell can be significantly increased by using two solar cells with different bandgaps $E_{g}$ in a row, i.e. a tandem solar cell (Figure 1a). The solar cell with the highest optical bandgap is in front (side of the sun), thus $E_{g 1}>E_{g 2}$. High-energy photons with an energy $h v>E_{g l}$ are absorbed by the first cell. The second cell, with a lower bandgap $E_{g 2}$, absorbs the low-energy photons with energy between $E_{g 1}$ and $E_{g 2}$. In this configuration, the photon energy is used more efficiently: the voltage at which electrical charge is collected in each subcell is closer to the energy of the photons absorbed in that subcell.

In the ideal configuration, the subcells are electrically separated. This is called the 4-terminal or stacked configuration (Figure 1a). However, this configuration is to date economical irrelevant. Indeed, experimental and commercial tandem solar cells are usually of the monolithic (integrated or 2terminal) type (Figure 1b). This means that the two cells are not only optically in series, but also electrically in series. This configuration will never reach an efficiency that is higher than that of a stacked tandem cell, because all single cells cannot operate at their optimal working point at the same time (unless they have an equal maximum-power current).

Because we want to investigate the relationship between on the one hand the energy levels of the subcells, and on the other hand the light harvesting potential of the configurations, we assume full absorption in each subcell (and consequently leaving the thickness of the subcells aside). We neglect interference and optical coupling of the subcells, thus overestimating the efficiency potential. Indeed, 
we only want to study the relative efficiency gain by using tandem cells instead of single-junction cells for indoor applications.

Figure 1. (a) A 4-terminal or stacked tandem solar cell: the first single cell absorbs photons with an energy $h v$ higher than $E_{g l}$. The second cell absorbs photons with an energy between $E_{g 1}$ and $E_{g 2}$. Photons with an energy below $E_{g 2}$ are not absorbed. The two subcells are electrically separated. (b) A monolithic or 2-terminal tandem solar cell: the single cells are electrically connected in series.

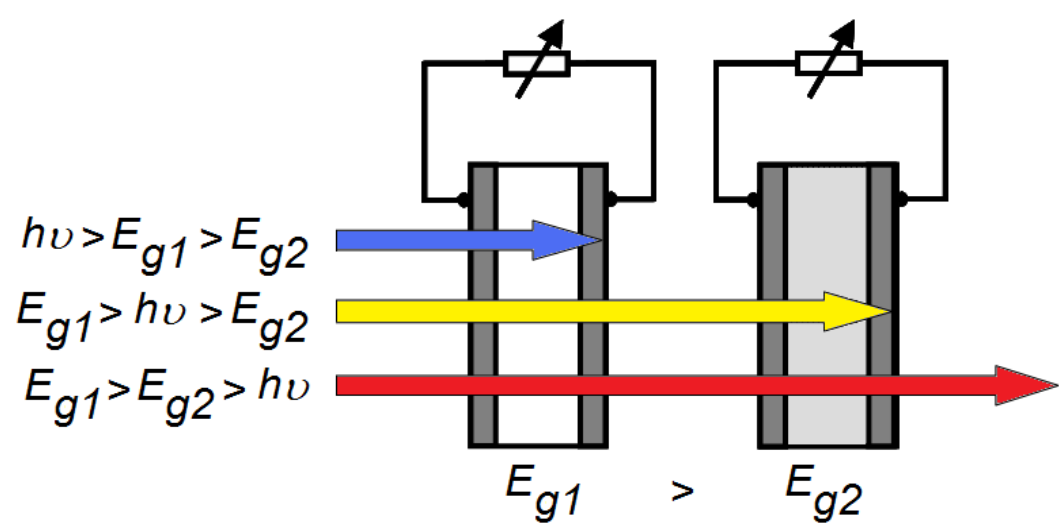

(a)

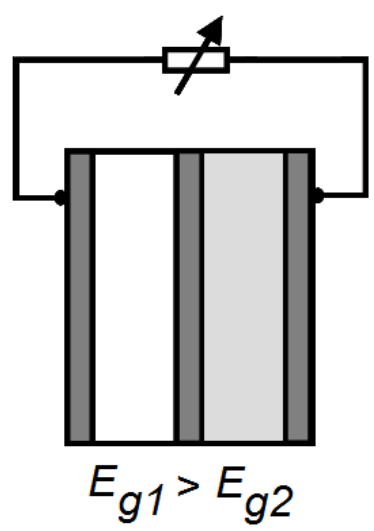

(b)

For our simulations, the following fundamental assumptions are made about the stacked tandem cell (Figure 1a):

- Every photon with an energy $h v$ higher than the bandgap $E_{g l}$ is absorbed by the first cell and leads to a useful energy $E_{g l}$. This assumption implies that each absorbed photon eventually leads to a free electron and a free hole, with an energy difference of $E_{g l}$ between them.

- Every photon with an energy $h v$ between $E_{g 1}$ and $E_{g 2}$ is absorbed by the second cell and leads to a useful energy $E_{g 2}$.

- Photons with an energy $h v$ lower than $E_{g 2}$ are fully transmitted.

The maximum efficiency $\eta_{\max }$ is therefore given by:

$$
\eta_{\max }=\frac{E_{g 1} \int_{E_{g 1}}^{\infty} N(E) d E+E_{g 2} \int_{E_{g 2}}^{E_{g 1}} N(E) d E}{\int_{0}^{\infty} E N(E) d E}, \text { with } E_{g 1}>E_{g 2}
$$

with $N(E)$ the incident photon flux. Note that the denominator is the total incident photon power density of the solar spectrum and does not depend on any bandgap. In this ideal scenario, the open circuit voltage $V_{o c}$ of the first and second subcell will be given by $E_{g l} / q$ and $E_{g 2} / q$ respectively (with $q$ the electric charge). The fill factor $F F$ of both subcells is assumed to equal unity, as well as the external quantum efficiency $E Q E$ of the first cell for wavelengths below the cut-off wavelength $\lambda_{\mathrm{g} 1}$ (corresponding with $E_{g l}$ ). The $E Q E$ of the second cell equals unity for wavelengths between cut-off wavelength $\lambda_{\mathrm{g} 1}$ and $\lambda_{\mathrm{g} 2}$ (corresponding with $E_{\mathrm{g} 2}$ ). Because these parameters are scalable, this idealization does not interfere with our goal to quantify the efficiency gain for tandem cells. 
In a monolithic or integrated tandem solar cell (Figure 1b), the individual cells are electrically connected in series. This means that the total voltage over the cell is the sum of the voltages over each individual cell, and thus equals the sum of the interface bandgaps of both single cells. Furthermore, the same current flows through both single cells. Hence, the maximum efficiency $\eta_{\max }$ for a monolithic organic tandem cell is given by:

$$
\eta_{\max }=\frac{\left(E_{g 1}+E_{g 2}\right) \cdot \min \left(\int_{E_{g 1}}^{\infty} N(E) d E, \int_{E_{g 2}}^{E_{g 1}} N(E) d E\right)}{\int_{0}^{\infty} E N(E) d E}, \text { with } E_{g 1}>E_{g 2}
$$

with $\min (x, y)$ the minimum of $x$ and $y$. The open circuit voltage $V_{o c}$ of the whole monolithic tandem cell will be given by $\left(E_{g l}+E_{g 2}\right) / q$, the fill factor $F F$ equals unity, as does the external quantum efficiency $E Q E$ for wavelengths below the cut-off wavelength $\lambda_{\mathrm{g} 2}$.

To calculate the performance of the single-junction, we make the same assumptions as the tandem cell, but with one cell, i.e. we use the same formula (1), but with $E_{g 2}=0$.

Figure 2. The spectral irradiance $E(\lambda)$ of typical artificial indoor light sources and the outdoor AM 1.5G spectrum as reference, all scaled to 500 lux. Please note that the spectra of the AM 1.5G and the incandescent lamp continue to higher wavelengths than $1000 \mathrm{~nm}$.

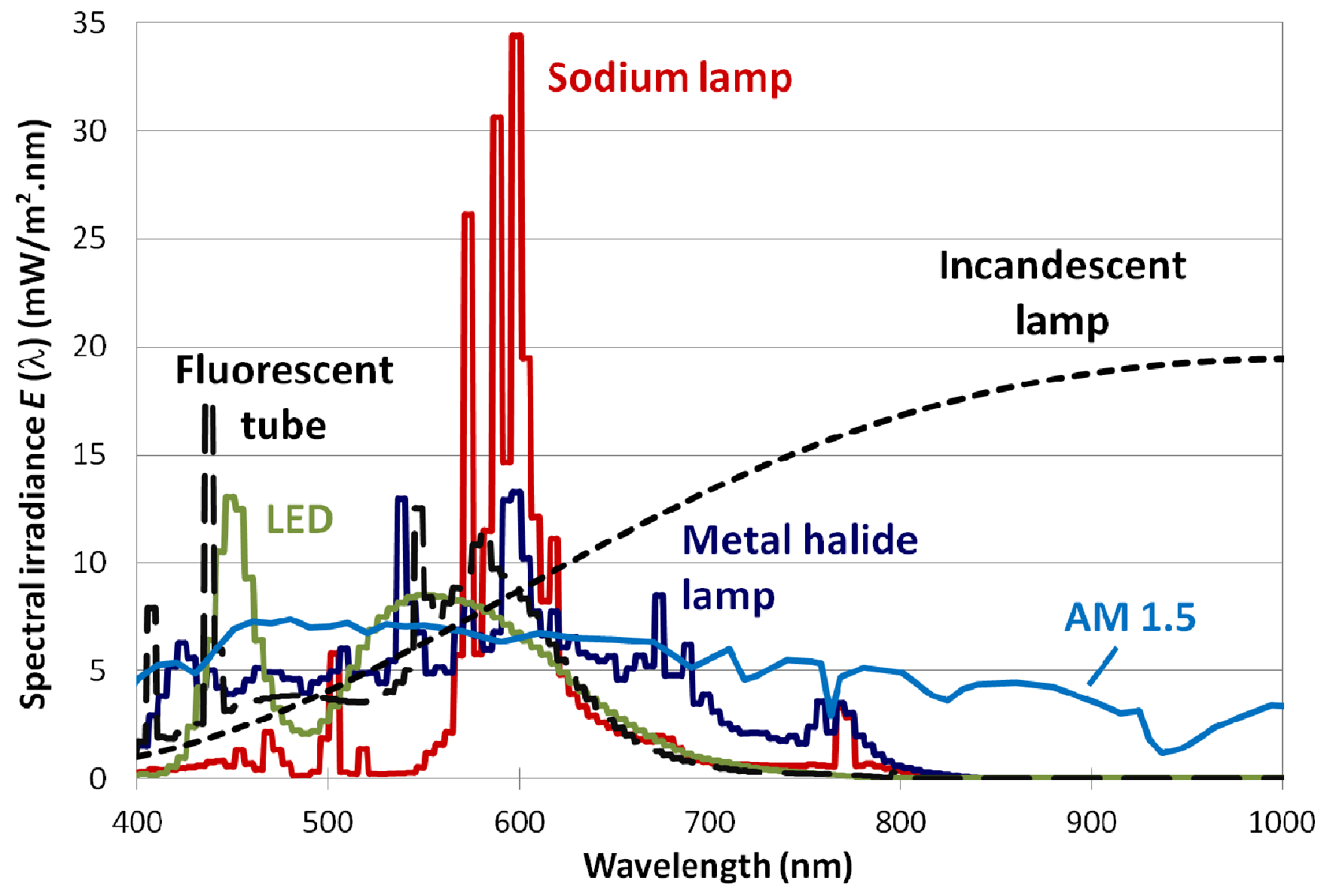


To study the potential of tandem solar cells for indoor applications, we consider the following indoor light sources:

- An incandescent lamp, represented by a black body on temperature $T=2856 \mathrm{~K}$.

- A cool white fluorescent lamp with a correlated color temperature of $4230 \mathrm{~K}$. They make up the majority of office illumination.

- Two types of high pressure discharge lamps which are commonly used in commercial and industrial environments: a sodium lamp and a metal halide lamp.

- A typical (cool) LED lamp.

We compare our results with the AM 1.5G spectrum (the outdoor standard spectrum) as reference [8]. If we want to compare the same lighting conditions, we have to scale all the light sources to an equal illumination for the human eye to obtain a correct comparison. We choose a value of 500 lux because it is recommended for general offices. For the goal of our paper, the value itself is not important, as long as it is the same for all light sources. To make a correct comparison possible, we also scale the AM 1.5 spectrum to 500 lux. The spectra can be found in Figure 2. We refer to [9, 10] for more details regarding the spectra.

\section{Results}

With the spectra and formulae above, we calculate the power conversion efficiency and the electrical power output of the single-junction and tandem solar cells under different indoor illuminations. We want to stress that the ideal efficiency from these calculations is not attainable in practice and the absolute value is only interesting from an academic point of view. However, the relative value of the single-junction compared to the tandem cell is relevant, as it describes the influence of one parameter only, namely the relative efficiency gain of adding an extra subcell to the configuration.

Figure 3 shows the maximum efficiency in this ideal scenario for a stacked and monolithic tandem cell with bandgaps $E_{g 1}$ and $E_{g 2}$ under the illumination of the AM 1.5 spectrum. A maximum efficiency of $65.2 \%$ and $63.5 \%$ is reached for a stacked and monolithic tandem cell respectively. As mentioned already, the efficiency of a monolithic configuration will never be higher than that of a stacked configuration. In comparison with a single-junction cell with an optimal bandgap of $1.1 \mathrm{eV}$, adding a second subcell results in a relative gain of about 1/3rd in power conversion efficiency [11]. For higher bandgaps, less photons are being absorbed from the solar spectrum, but the useful output energy of each absorbed photon is higher. Hence, there is an optimum for each bandgap. This maximum occurs for the stacked and monolithic tandem cell at a configuration $\left(E_{g 1}, E_{g 2}\right)$ of $(1.5 \mathrm{eV}, 0.7 \mathrm{eV})$ and $(1.6 \mathrm{eV}$, $0.9 \mathrm{eV}$ ) respectively. 
Figure 3. The maximum efficiency for a stacked (left) and monolithic (right) tandem solar cell with bandgaps $E_{g 1}$ and $E_{g 2}$ under illumination of the AM 1.5 spectrum (top), an incandescent lamp (middle) and a fluorescent tube (bottom).

\section{Stacked}
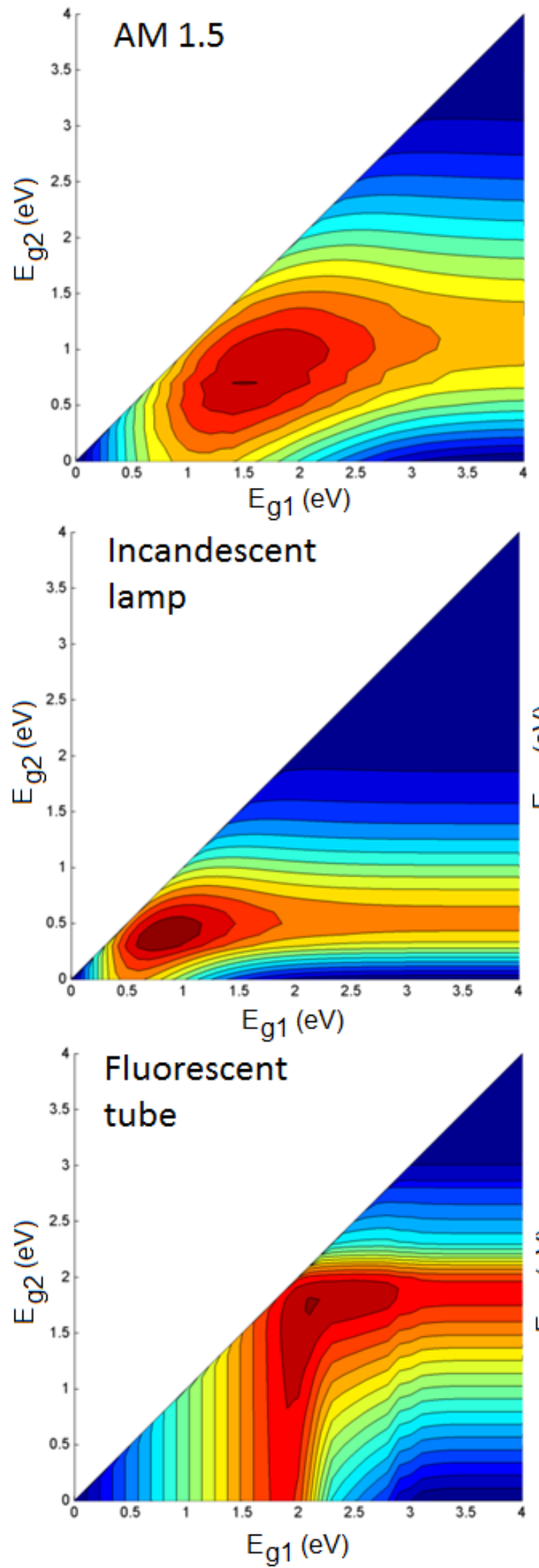

\section{Monolithic}
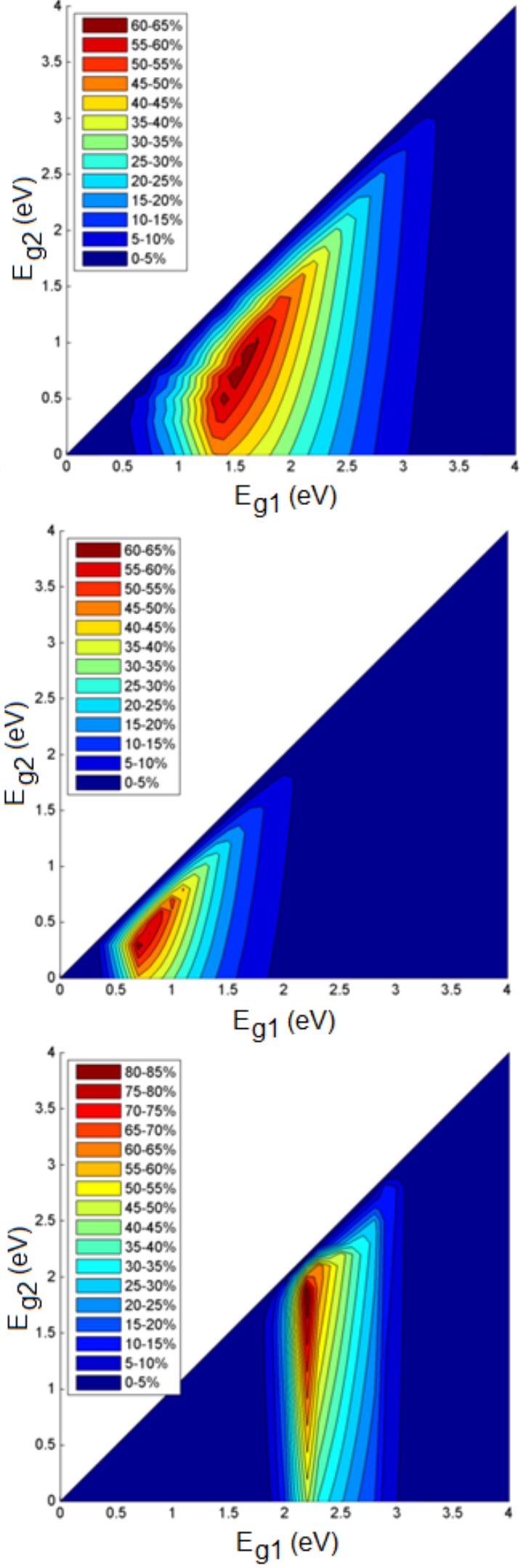
The requirements for a close to optimal configuration of the stacked tandem cell are quite broad, permitting that the values of the bandgaps for optimal cells are not that strict. This is not the case for the monolithic configuration; especially the value of the bandgap $E_{g l}$ of the first subcell is more critical than for a stacked cell. The current extracted from the monolithic tandem cell is almost equal to the photocurrent of the subcell that generates the lowest current. If one of the two cells generates much more current than the other cell, the excess of charge carriers cannot recombine at the intermediate contact between the subcells. This will cause a charging at the intermediate contact and will partially compensate the built-in voltage across the other cell until the current of both subcells matches. This explains the inferior power conversion of monolithic cells for non-optimal bandgap configurations. Current matching is therefore necessary in a monolithic configuration. We want to stress that this effect is not implemented in the model presented in this paper.

Figure 4. The optimal bandgaps $E_{g 1}$ and $E_{g 2}$ in $\mathrm{eV}$ of the subcells of a stacked and a monolithic tandem solar cell under different illuminations.

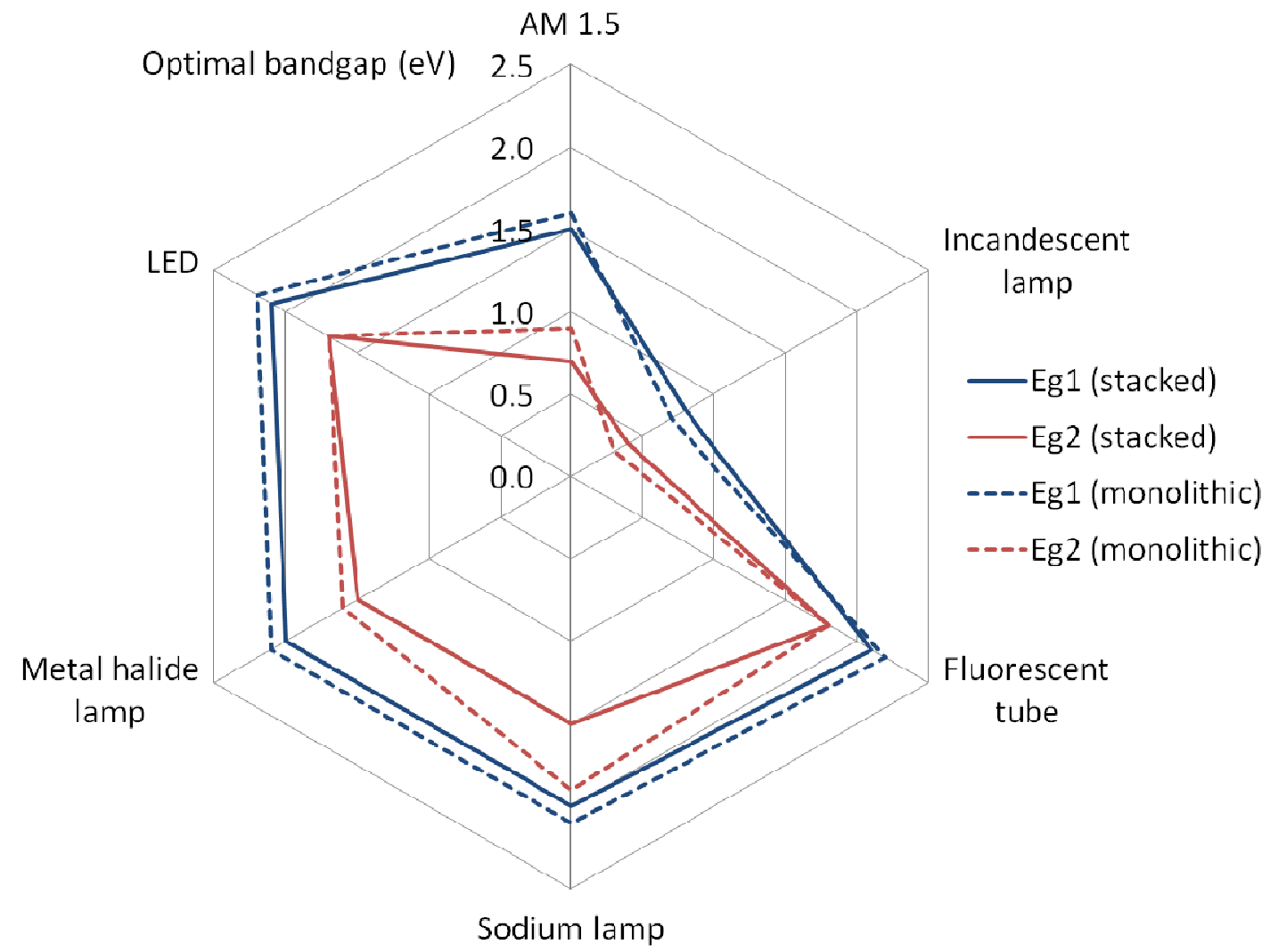

Figure 3 also shows the simulation results if we illuminate the photovoltaic tandem cell with the incandescent lamp and the fluorescent tube of Figure 2. We notice the same qualitative difference between the stacked and monolithic configurations. Please note that one may not compare the absolute value of the efficiencies with these artificial light sources to the efficiency of the AM 1.5 spectrum because the denominator in formulae (1) and (2) are different. As already mentioned, it will be the relative differences between the single and tandem junctions that are relevant.

The optimal bandgap of the tandem configuration (Figures 3 and 4) shifts to lower values for the incandescent lamp and to higher values for the modern light sources (fluorescent tube, sodium lamp, 
metal halide lamp and LED lamp), compared to the outdoor AM 1.5 reference. The reason is that the incandescent lamp was not optimized to the visible light for the human eye. The other, more modern, light sources are much more energy efficient than the incandescent lamp. Indeed, modern light sources emit light mostly within the visible region, from 380 (violet) to $780 \mathrm{~nm}$ (red), which make them very energy efficient: emitting light within the visible spectrum is the primary goal of light sources. However, e.g. a mono crystalline Si cell can absorb light to $1200 \mathrm{~nm}$. The incandescent lamp (and to a lesser extent the AM 1.5 spectrum) emit a large part of their radiation in the longer (non-visible) wavelength region. This results in lower optimal bandgaps than for the modern light sources which only emit to about $800 \mathrm{~nm}$.

Figure 5. The ratio of the calculated electrical power output of a stacked and monolithic tandem configuration to the single-junction configuration for the different illuminations.

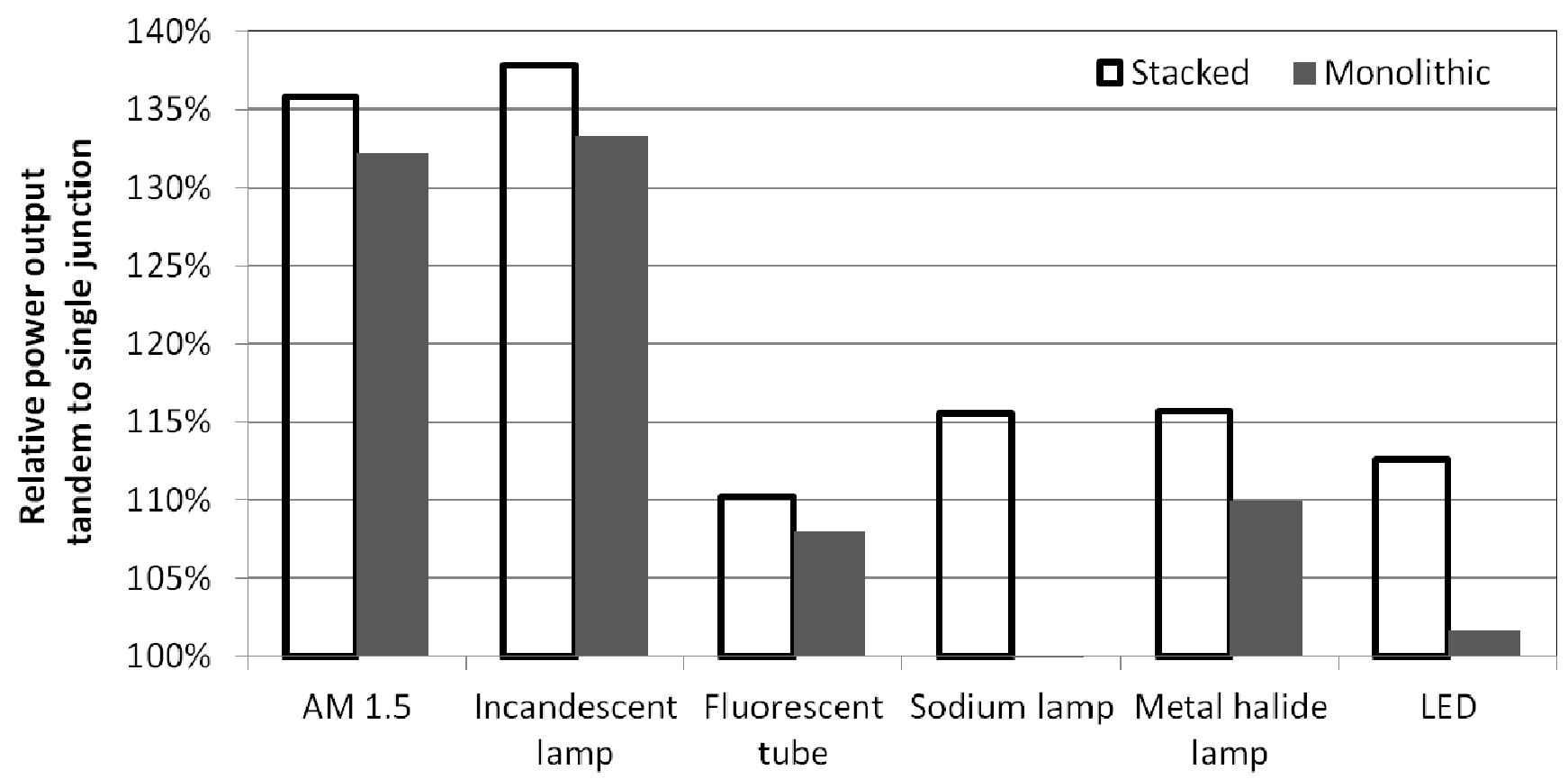

Figure 5 shows the ratio of the calculated electrical power output of the tandem configuration to the single-junction configuration for the light sources at the optimal bandgaps. We again notice the difference between the modern light sources (which mainly emit light within the visible region) and the incandescent lamp and AM 1.5 spectrum (which also emit light in the non-visible region). Using a tandem cell instead of a single- junction can improve the electrical output of the solar cell with about one third under AM 1.5 illumination and incandescent light. The improvement for the modern light sources is maximum $15 \%$. But the stacked configuration is to date economical irrelevant. Nowadays, experimental and commercial tandem solar cells are usually of the monolithic type. If we only consider this type, the maximum efficiency gain for adding an extra subcell to a single-junction is maximum 10 \% (for the metal halide lamp) and even negligible for the LED and sodium lamp. Taken into account the fact that indoor solar cells will mainly be used for cheap applications (wireless communication networks, router nodes, sensor networks,...), the extra cost of producing a tandem configuration will probably not be worth the limited efficiency gain. 


\section{Conclusions}

We compared the power output of tandem solar cells with single-junctions as reference for typical artificial indoor light sources. We studied the influence of the bandgaps of a tandem solar cell for typical artificial indoor light sources and found that there is a significant difference between the modern light sources (which mainly emit light within the visible region) and the incandescent lamp and AM 1.5 spectrum (which also emit light in the non-visible region). We not only considered the usual monolithic configuration, but also the stacked set-up. By determining the relative efficiency gain compared to single-junctions, we demonstrated the limited usefulness of tandem solar cells for indoor applications.

\section{Conflicts of Interest}

The authors declare no conflict of interest.

\section{References and Notes}

1. Reich, N.H.; Veefkind, M.; van Sark, W.G.J.H.M.; Alsema, E.A.; Turkenburg, W.C.; Silvester, S. A solar powered wireless computer mouse: Industrial design concepts. Solar Energy 2009, 83, 202-210.

2. Girish, T.E. Some suggestions for photovoltaic power generation using artificial light illumination. Solar Energy Materials and Solar Cells 2006, 90, 2569-2571.

3. Minnaert, B.; Veelaert, P., Efficiency simulations of thin film chalcogenide photovoltaic cells for different indoor lighting conditions. Thin Solid Films 2011, 519, 7537-7540.

4. Randall, J.F.; Jacot, J., Is AM1.5 applicable in practice? Modelling eight photovoltaic materials with respect to light intensity and two spectra. Renewable Energy 2003, 28, 1851-1864.

5. Randall, J.F; Jacot, J. The performance and modelling of 8 photovoltaic materials under variable light intensity and spectra. Proceedings of the World Renewable Energy Congress VII \& Expo, Cologne, Germany, 29 June - 5 July 2002.

6. Steim, R.; Ameri, T.; Schilinsky, P.; Waldauf, C.; Dennler, G.; Scharber, M.; Brabec, C.J., Organic photovoltaics for low light applications. Solar Energy Materials and Solar Cells 2011, 95, 3256-3261.

7. Monokroussos, C.; Bliss, M.; Qiu, Y.N.; Hibberd, C.J.; Betts, T.R.; Tiwari, A.N.; Gottschalg, R., Effects of spectrum on the power rating of amorphous silicon photovoltaic devices. Progress in Photovoltaics 2011, 19, 640-648.

8. International standard, IEC 60904-3, edition 2, April 2008, photovoltaic devices-part 3: Measurement principles for terrestrial photovoltaic (PV) solar devices with reference spectral irradiance data, ISBN 2-8318- 9705-x. International Electrotechnical Commission: 2008.

9. International Commission on Illumination. CIE Technical Report Colorimetry, CIE 15:2004 (3rd Edition); International Commission on Illumination: 2004.

10. Minnaert, B.; Veelaert, P., Efficiency simulations of thin film chalcogenide photovoltaic cells for different indoor lighting conditions. Thin Solid Films 2011, 519, 7537-7540. 
11. Minnaert, B.; Burgelman, M., Efficiency potential of organic bulk heterojunction solar cells. Progress in Photovoltaics 2007, 15, 741-748.

(C) 2014 by the authors; licensee MDPI, Basel, Switzerland. This article is an open access article distributed under the terms and conditions of the Creative Commons Attribution license (http://creativecommons.org/licenses/by/3.0/). 